\title{
Robust Beamforming Problem in Cognitive One-way Relay Networks
}

\author{
Hongyan Cai and Wenbo Wang \\ School of Information and Communication Engineering, \\ Beijing University of Posts and Telecommunications \\ Address: Beijing University of Posts and Telecommunications, \\ No 10, Xitucheng Road, Haidian District, Beijing, PRC \\ hy_cai75@163.com
}

\begin{abstract}
The paper investigates the problem of a distributed robust relay beamforming in cognitive one-way relay networks with imperfect channel state information. We aim to the minimum the transmit power of relays, satisfying the SNR (signal-to-noise ratio) of the secondary users high a threshold and keeping the interference at primary users below an acceptable threshold. Because of the robust identity the optimal problem is a non-convex problem which is hard to be solved. In this paper we transform the non-convex problem into a convex one which can be solved efficiently by exploiting S-procedure and Schur complement theorem and relaxing the rank-one constrain. Then the robust beamforming vector can be obtained. Simulation results show that the proposed algorithm can solve the minimum problem with robust features efficiently and obtain satisfactory results.
\end{abstract}

Keyword: Relay Networks, Robust, Cognitive Networks; SDP; Rank-one

\section{Introduction}

Cognitive relay network (CRN) combines both parts: (1) The cognitive radio network which is an effective way to improve the spectrum utilization; (2) The relay network which is a powerful technology that combats signal fading due to multipath propagation in a wireless medium. The concept of cognitive radio (CR) is first proposed by Dr. Mitola in 1999[1].CRN is a popular method in improving the performance of the secondary users (SUs) and guaranteeing the QoS of the primary users (PUs).Now most of the research works is based on the known ideal channel state information (CSI) which is hard to obtain in practice, however, nonideal CSI will affect the performance of the network seriously[2].So more and more scholars are interested in the robust beamforming algorithms in the non-cognitive or cognitive relay network and have published many papers about this topic[3-4].

In this paper, we focus on the performance of the robust cooperative beamforming in the cognitive relay network and minimize the total transmit power of the relay, while emphasize the interference at the primary receiver.

\section{System Model}

We study a cognitive network $(\mathrm{CN})[1]$ which includes two parts as Figure 1: primary network (PN) with the primary transmitter (PT) and the primary destination (PD), and secondary network (SN) with the secondary transmitter (ST), N relays $\left\{S R_{i}\right\}_{i=1}^{N}$ and the secondary destination (SD). All nodes in network have been configured with one antenna. We assume that there is no direct link between the sources and the destinations, the help of relays is necessary to establish the communication link. Here we use AF protocol. The dotted lines in Figure 1 represent the interference channels between different transmitters 
and receivers. We assume ST is far from PD and PT is far from SD, so the interference between them is ignored and assume a flat fading scenario. We denote the channel between ST and $S R_{i}$ (the $i$ th relay) as $h_{S R_{i}} \hat{I} £$, the channel between PT and $S R_{i}$ is $g_{P R_{i}} \hat{I} £$, the channel between $S R_{i}$ and $\mathrm{SD}$ as $h_{R_{i} d} \hat{I} £$, and the channel between $S R_{i}$ and PD as $g_{R_{i} d} \hat{\mathrm{I}} £, i=1, \mathrm{~L}, N$. The channel between PT and PD denote as $h_{P D} \hat{I} £ .[5][6]$

The transmitter in the CRN divides the process into two sub-processes. In the first sub-process, PT transmits message $s 1$ to PD and ST transmits message $x$ to SR. In the second sub-process, PT transmits message $s 2$ to PD and SR retransmit the received signal to SD. We herein use a two-step BF protocol. To simplify the analysis, we assume that $x, s 1, s 2$ are independent to each other, and $E\left[|x|^{2}\right]=1, E\left[|s 1|^{2}\right]=1, E\left[|s 2|^{2}\right]=1$.

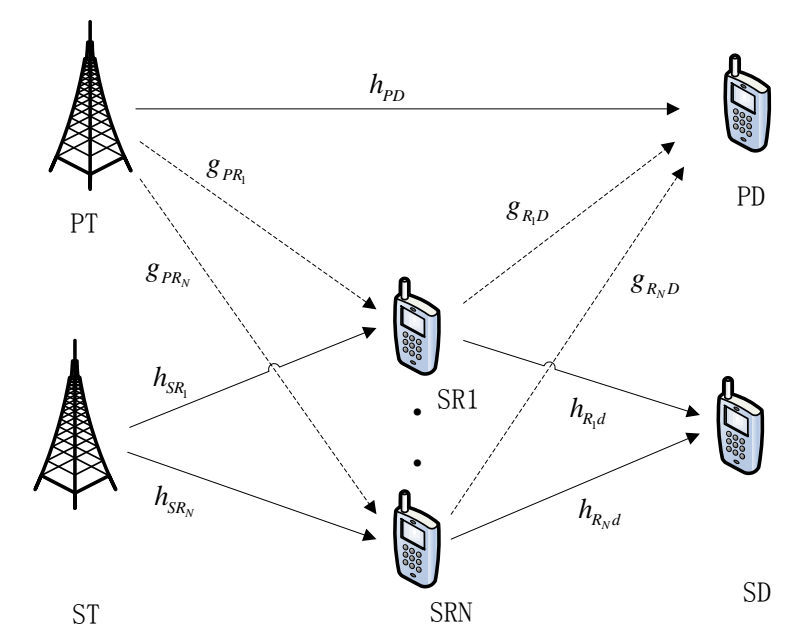

Figure 1. System Model

During the MAC, the transmit power of PT and ST are $P_{P}$ and $P_{S}$ respectively. PT transmits message $s 1$ to PD and ST transmits message $x$ to SR. Then the received signal at $i$ th relay is given as follows:

$$
r_{i}=\sqrt{P_{S}} h_{S R_{i}} x+\sqrt{P_{P}} g_{P R_{i}} s_{1}+n_{R_{i}}
$$

where, $n_{R_{i}} \square N\left(0, N_{R}\right)$ represents the complex Gaussian noise at the ${ }_{i}$ th relay.

During the BC, PT transmits message $s 2$ to PD with the same power $P_{P}$ as the MAC. The retransmit signal $y_{i}$ of the $i$ th relay is

$$
y_{i}=w_{i} r_{i}
$$

Where, $w_{i} \hat{I} £$ is the complex beamforming weight. Then the received signals at PD and SD can be written as:

$$
\begin{aligned}
& y_{p}=\sqrt{P_{P}} h_{P D} s_{2}+\stackrel{a}{i=1}_{N}^{N} w_{i} g_{R_{i} D} r_{i}+n_{D} \\
& y_{s}=\stackrel{\mathrm{a}}{i=1}^{N} w_{i} h_{R_{i} D} r_{i}+n_{d}
\end{aligned}
$$

where, $n_{D} \square N\left(0, N_{D}\right), n_{d} \square N\left(0, N_{d}\right)$ represent the complex Gaussian noise at PD and SD.

Substituting (1) into (3) and (4) we can reorganize them as shown in (5) and (6) 


$$
\begin{aligned}
& y_{p}=\sqrt{P_{P}} h_{P D} s_{2}+\stackrel{N}{\stackrel{\circ}{a}} w_{i=1} g_{R_{i} D}\left(\sqrt{P_{S}} h_{S R_{i}} x+\sqrt{P_{P}} g_{P R_{i}} s_{1}+n_{R_{i}}\right)+n_{D}
\end{aligned}
$$



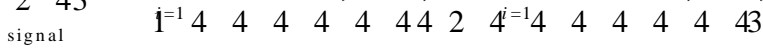

$$
\begin{aligned}
& +\stackrel{N}{\mathrm{a}} \quad w_{i} g_{R_{i} D} n_{R_{i}}+n_{D} \\
& \mathrm{i}^{=1} 4 \begin{array}{lllll}
4 & 2 & 4 & 4 & 3
\end{array} \\
& y_{s}=\stackrel{\AA}{i=1}^{N} w_{i} h_{R_{i} d}\left(\sqrt{P_{S}} h_{S R_{i}} x+\sqrt{P_{P}} g_{P R_{i}} s_{1}+n_{R_{i}}\right)+n_{d} \\
& =\stackrel{N}{\mathrm{a}} \sqrt{P_{S}} w_{i} h_{R_{i} d} h_{S R_{i}} x \\
& \mathrm{i}^{1=1} 4 \begin{array}{llllll}
4 & 2 & 4 & 4 & 3
\end{array} \\
& \text { signal, } \mathrm{s}_{T} \\
& +\stackrel{N}{\mathrm{a}} \sqrt{P_{P}} w_{i} h_{R_{i} d} g_{P R_{i}} s_{1}+\stackrel{N}{\mathrm{a}} w_{i} h_{R_{i} d} n_{R_{i}}+n_{d} \\
& \mathrm{i}^{=1} 4 \quad 44 \begin{array}{lllllllllll} 
& 4 & 4 & 4 & \mathcal{B} & \mathrm{j}^{=1} 4 & 4 & 2 & 4 & 4 & 3
\end{array} \\
& \begin{array}{lllllllllll}
1 & 4 & 4^{\text {nt }} 4^{\text {fergnce }} 4 & 4 & 2 & 4 & 4 & 4 & \text { goisq } & 4 & 3
\end{array}
\end{aligned}
$$

During the BC, the received signal of PD is $s_{2}$, not $s_{1}$, so $s_{1}$ of (6) is interference.

The paper minimizes the total transmit power of the relay, while emphasizes the interference at the primary receiver.

\section{Robust Distributed Beamforming}

The total transmitted power of relays is:

$$
\begin{aligned}
P_{T} & =\sum_{i=1}^{N} E\left\{\left|y_{i}\right|^{2}\right\}=\sum_{i=1}^{N}\left|w_{i}\right|^{2} E\left\{\left|r_{i}\right|^{2}\right\}=w^{H} D w \\
D= & \mathrm{d} \text { i a } \mathrm{g} P\left\{h_{S R_{1}}{ }^{2} P_{P}+{ }_{P R}\left|\mathrm{~g}_{1}^{2} P_{S} h_{S R},{ }_{2}^{2}\right|\right. \\
& +P_{P}\left|\mathrm{~g}_{R_{2}}{ }^{2}\right| \mathrm{L}, P_{s},\left.h_{S R}\right|^{2} P_{P}+_{P R_{N}}\left|\mathrm{~g}^{2} N\right|_{R} \mathbf{I}
\end{aligned}
$$

The disturbed power of PD is:

$$
\begin{aligned}
& Y_{p}=E\left\{\left|n_{P}\right|^{2}\right\} \\
& =E\left\{\left|\sum_{i=1}^{N} \sqrt{P_{P}} w_{i} g_{R_{i} D} g_{P R_{i}} s_{1}+\sum_{i=1}^{N} \sqrt{P_{S}} w_{i} g_{R_{i} D} h_{S R_{i}} x\right|^{2}\right\} \\
& =P_{P} g_{R D}^{H}\left(w \square g_{P R}\right)\left(w \square g_{P R}\right)^{H} g_{R D}+P_{S} g_{R D}^{H}\left(w \square h_{S R}\right)\left(w \square h_{S R}\right)^{H} g_{R D} \\
& =g_{R D}^{H}\left[P_{P}\left(w \square g_{P R}\right)\left(w \square g_{P R}\right)^{H}+P_{S}\left(w \square h_{S R}\right)\left(w \square h_{S R}\right)^{H}\right] g_{R D}
\end{aligned}
$$

$S_{P}, N_{P}$ are the received signal power and the noise power of SD respectively, so we have

$$
\begin{aligned}
& S_{P}=E\left\{\left|s_{T}\right|^{2}\right\}=E\left\{\left|\underset{i=1}{\mathrm{a}} \sqrt{P_{S}} w_{i} h_{R_{i} d} h_{S R_{i}} x\right|^{2}\right\} \\
& =P_{S} h_{R d}^{H}\left(w \mathrm{e} h_{S R}\right)\left(w \mathrm{e} h_{S R}\right)^{H} h_{R d} \\
& N_{P}=E\left\{\left|n_{T}\right|^{2}\right\} \\
& =E\left\{\left|a_{i=1}^{N} \sqrt{P_{P}} w_{i} h_{R_{i} d} g_{P R_{i}} s_{1}+\underset{i=1}{\mathrm{a}} w_{i} h_{R_{i} d} n_{R_{i}}+n_{d}\right|^{2}\right\} \\
& =P_{P} h_{R d}^{H}\left[\left(w \mathrm{e} g_{P R}\right)\left(\begin{array}{ll}
w & \mathrm{e} \\
g_{P R}
\end{array}\right)^{H}+N_{R} w w^{H}\right] h_{R d}+N_{d}
\end{aligned}
$$


where, $w=\left(w_{1}, w_{2}, \mathrm{~L}, w_{N}\right)^{H}, g_{R D}=\left(g_{R_{1} D}, g_{R_{2} D}, \mathrm{~L}, g_{R_{N} D}\right), g_{P R}=\left(g_{P R_{1}}, g_{P R_{2}}, \mathrm{~L}, g_{P R_{N}}\right)$

$h_{S R}=\left(h_{S R_{1}}, h_{S R_{2}}, \mathrm{~L}, h_{S R_{N}}\right), h_{R d}=\left(h_{R_{1} d}, h_{R_{2} d}, \mathrm{~L}, h_{R_{N} d}\right)$.

The SNR of SD is defined as ${ }_{S I N R}=\frac{S_{P}}{N_{P}}$.

In the section, our aim is to obtain the optimal robust beamforming weight coefficient and minimum transmission power of the relays. But we must ensure the GoS of the primary network users, which is that the interference is less than the threshold $\delta$ given in advance for primary network users, moreover, we must ensure that the SNR of the secondary network users are larger than some threshold $\gamma$.

So the optimal model can write as following:

$$
\begin{aligned}
& \min w^{H} D w \\
& \text { s.t. } \frac{P_{S} h_{R d}^{H}\left(w \text { e } h_{S R}\right)\left(w \text { e } h_{S R}\right)^{H} h_{R d}}{h_{R d}^{H}\left[P_{P}\left(w \text { e } g_{P R}\right)\left(w \text { e } g_{P R}\right)^{H}+N_{R} w w^{H}\right] h_{R d}+N_{d}}{ }^{3} g \\
& g_{R D}^{H}\left[P_{P}\left(w \mathrm{e} \quad g_{P R}\right)\left(w \text { e } g_{P R}\right)^{H}+P_{S}\left(w \text { e } h_{S R}\right)\left(w \text { e } h_{S R}\right)^{H}\right] g_{R D} £ d .
\end{aligned}
$$

In this paper, we assume the central processor is placed the inside of relays or the near of relays for searching CSI vector $h_{S R_{i}}, g_{P R_{i}}, h_{R_{i} d}$ and $g_{R_{i} d}, i=1 \mathrm{~L} N$. Because there is quantization error and feedback time-delay error in the actual transmission, ideal CSI vector $h_{R_{i} d}, g_{R_{i} D}, i=1, \mathrm{~L}, N$ can't be obtained, CSI error model may be expressed as:

$$
h_{R d}=\bar{h}_{R d}+\mathrm{D} h_{R d}, g_{R D}=\bar{g}_{R D}+\mathrm{D} g_{R D},
$$

where, $\bar{h}_{R d}, \bar{g}_{R D}$ are the observed channel vector of $h_{R d}$ and $g_{R D}$ respectively, the error vector $\mathrm{D} h_{R d}, \mathrm{D} g_{R D}$ are bounded:

$$
\begin{aligned}
& \mathrm{D} h_{R d}^{H} \mathrm{D} h_{R d} £ M r \\
& \mathrm{D} g_{R D}^{H} \mathrm{D} g_{R D} £ M r
\end{aligned}
$$

Because of $(a$ e $b)(a \text { e } b)^{H}=a a^{H}$ e $b b^{H}$ [7], so the first constraint of optimization (11) can be rewritten as:

$$
\frac{P_{S} h_{R d}^{H}\left(w \mathrm{e} h_{S R}\right)\left(w \mathrm{e} h_{S R}\right)^{H} h_{R d}}{h_{R d}^{H}\left[P_{P}\left(w \mathrm{e} g_{P R}\right)\left(w \mathrm{e} g_{P R}\right)^{H}+N_{R} w w^{H}\right] h_{R d}+N_{d}}{ }^{3} r
$$

And (14) is equivalent to (15)

$$
h_{R d}^{H}\left[\left(P_{S} h_{S R} h_{S R}^{H}-r P_{P} g_{P R} g_{P R}^{H}-r N_{R} I\right) \mathrm{e} w w^{H}\right] h_{R d}{ }^{3} r N_{d}
$$

The second constraint of (11) can rewritten as

$$
g_{R D}^{H}\left[P_{P}\left(w \mathrm{e} \quad g_{P R}\right)\left(w \mathrm{e} \quad g_{P R}\right)^{H}+P_{S}\left(w \mathrm{e} \quad h_{S R}\right)\left(w \mathrm{e} h_{S R}\right)^{H}\right] g_{R D} £ d .
$$

And (16) is equivalent to (17)

$$
g_{R D}^{H}\left[\left(P_{P} g_{P R} g_{P R}^{H}-P_{S} h_{S R} h_{S R}^{H}\right) \text { e } w w^{H}\right] g_{R D} £ d .
$$

The optimization (11) becomes into the following form equivalently: 


$$
\begin{aligned}
& \min w^{H} D w \\
& \text { s.t. } h_{R d}^{H}\left[\left(P_{S} h_{S R} h_{s R}^{H}-r P_{P} g_{P R} g_{P R}^{H}-r N_{R} I\right) \mathrm{e} w w^{H}\right] h_{R d}{ }^{3} r N_{d}, \\
& \quad g_{R D}^{H}\left[\left(P_{P} g_{P R} g_{P R}^{H}-P_{S} h_{S R} h_{s R}^{H}\right) \mathrm{e} w w^{H}\right] g_{R D} £ d, \\
& \quad \mathrm{D} h_{R d}^{H} \mathrm{D} h_{R d} £ M r \\
& \quad \mathrm{D} g_{R D}^{H} \mathrm{D} g_{R D} £ M r .
\end{aligned}
$$

Since $\mathrm{D} h_{R d}, \mathrm{D} g_{R D}$ are continuous, there are infinite realization of $\mathrm{D} h_{R d}$ and $\mathrm{D} g_{R D}$,which make the problem unsolvable. To proceed, we will introduce a theorem S-procedure as follow.

Lemma 1 Let $P$ and $Q$ are both symmetric matrix, there is a vector $u_{0}$ which satisfies $\left(u_{0}\right)^{T} P\left(u_{0}\right)>0$, then " $u^{T} P u^{3} 0 \mathrm{P} \quad u^{T} Q u^{3} 0$ " iff “ $\$ l l^{3} 0$, s.t.

$Q$ f_l $l P$ ’.[8-9]

We will turn infinite constraints into finite constraints by Lemma 1.

Let $Q=\left(P_{S} h_{S R} h_{S R}^{H}-r P_{P} g_{P R} g_{P R}^{H}-r N_{R} I\right) \mathrm{e} w w^{H}, T=\left(P_{P} g_{P R} g_{P R}^{H}-P_{S} h_{S R} h_{S R}^{H}\right) \mathrm{e} w w^{H}$, Then, the first constraint of(18)can translate to

$$
\begin{aligned}
& \left(\bar{h}_{R d}+\mathrm{D} h_{R d}\right)^{H} Q\left(\bar{h}_{R d}+\mathrm{D} h_{R d}\right)-r N_{d}{ }^{3} 0
\end{aligned}
$$

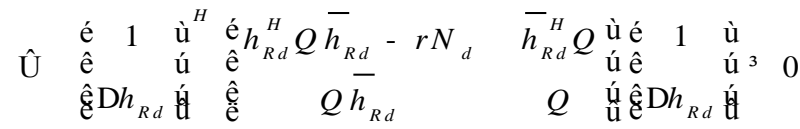

the second constraint of (18) can translate to:

$$
\begin{aligned}
& \left.d-\left(\bar{g}_{R D}+\mathrm{D} g_{R D}\right)^{H} T \quad \bar{g}_{R D}+\mathrm{D} g_{R D}{ }^{3}\right) \quad 0
\end{aligned}
$$

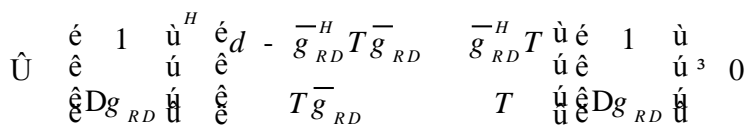

the third constraint of (18) can translate to

$$
\begin{aligned}
& \mathrm{D} h_{R d}^{H} \mathrm{D} h_{R d} £ M r
\end{aligned}
$$

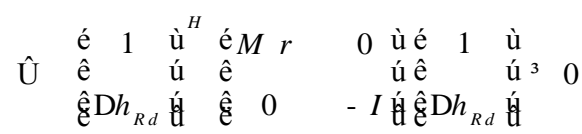

the forth constraint of (18) can translate to

$$
\begin{aligned}
& \mathrm{D} g_{R D}^{H} \mathrm{D} g_{R D} £ M r
\end{aligned}
$$

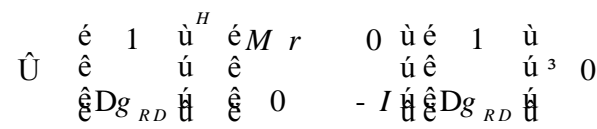

We apply S-procedure to(14)and(16),(15)and(17), then we can obtain the following form:

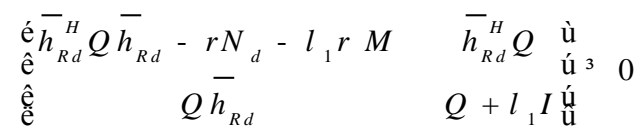

$$
\begin{aligned}
& { }_{\hat{e}}^{\text {é }} d-l_{2} r M-\bar{g}_{R D}^{H} T \bar{g}_{R D} \quad \bar{g}_{R D}^{H} T \text { ù }{ }^{3}{ }^{3} 0
\end{aligned}
$$

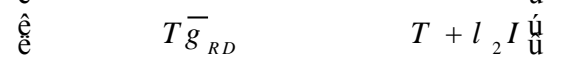

Let $W=w w^{H}, \operatorname{rank}(W)=1$, rewrite the optimization problem(18) 


$$
\begin{aligned}
& \min D \cdot W
\end{aligned}
$$

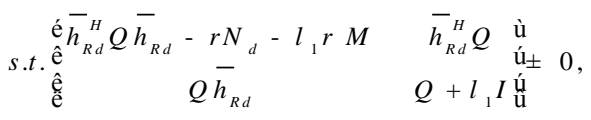

$$
\begin{aligned}
& \begin{array}{l}
\text { é } d-l_{2} r M-\bar{g}_{R D}^{H} T \bar{g}_{R D} \quad \bar{g}_{R D}^{H} T \text { ù } \\
\hat{\mathrm{e}} \\
\hat{\mathrm{e}}
\end{array} \\
& l_{1}, l_{2}{ }^{3} 0 \text {, } \\
& W \pm 0 \text {. } \\
& \text { Rand }(W)=1
\end{aligned}
$$

Due to the rank-one constraint, the optimization problem (25) is hard to be solved. Therefore we resort to relaxing (25) by deleting the rank-one constraint $R \operatorname{ank}(W)=1$, [8-9] namely

$$
\begin{aligned}
& \text { m in } D \cdot W
\end{aligned}
$$

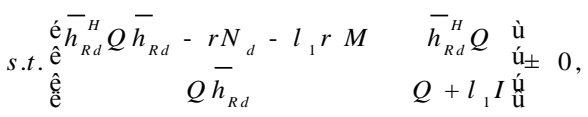

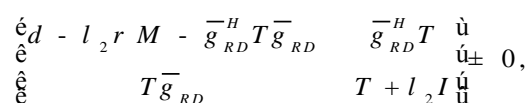

$$
\begin{aligned}
& l_{1}, l_{2}{ }^{3} 0 \text {, } \\
& W \pm 0 .
\end{aligned}
$$

We can verify that (26) is a semi-definite programming (SDP) problem easily and can obtain the optimal solution by MATLAB. If the optimal solution $W^{*}$ of (26) is rank-one, the optimal solution $w^{*}$ of (25) can be obtained by using eigenvalue decomposition. But if $W^{*}$ is not rank-one, we must resort to some other ways. Now, randomization and eigenvector approximation method is often used to find the suitable solution [10]. In this paper, we will choose the randomization method for the optimal solution $w^{*}$ of (25).

\section{Simulation results}

In this section, these simulations are based on independent Rayleigh flat-fading channels. The noise of is $1, P_{1}=P_{2}=P T=10 d B, d=5 d B$.Fig 2 depicts that the total transmitter power of relays become large significantly with the increase of SNRs of secondary network when the interference temperature is $-2 \mathrm{~dB}$ or $2 \mathrm{~dB}$, and we obtain the same conclusion in the case of simulating some other interference temperature. Simultaneously, we also find that the total transmit power of relays increase obviously when the SNR of the cognitive users are larger requested. So we need a trade-off in practice. In Fig3, we can see that the total transmitter power of relays decreases significantly with the increase of the relay number. Moreover, we assert that we can decrease the total transmit power of relays when increasing the number of relay. When the number of relays is larger than 25 , the total transmit power decrease no obviously. In practice, we should choose the number of the relay properly in considering the cost of relays. 




Figure 2. The SNR of the Cognitive Users vs Total Relay Transmit Power

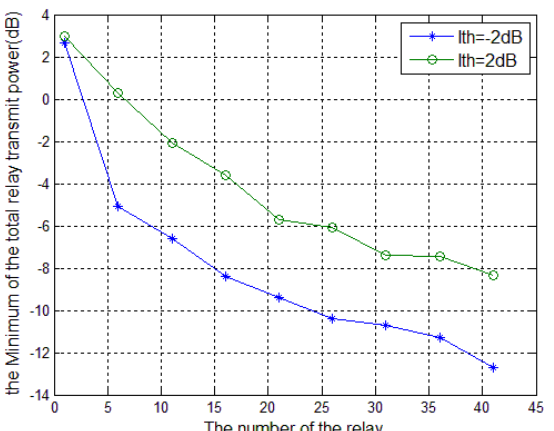

Figure 3. The Number of the Relay vs Total Relay Transmit Power

\section{Conclusions}

In this paper, we proposed the robust relay beamforming for CRN using semi-definite programming method. The Cognitive radio system have a primary network (PN) with the primary transmitter (PT) and the primary destination (PD), have a secondary network (SN) with the secondary transmitter (ST), N relays and the secondary destination (SD).We design a robust optimal model that minimizes the total power of relays, while emphasize the interference at the primary receiver. We transform the non-convex robust optima problem into a convex one which can be solved efficiently by exploiting S-procedure and relax the rank-one constrain. Simulation results show that the proposed algorithm can solve the minimum problem with robust features efficiently and obtain satisfactory results.

\section{Acknowledgements}

This work is supported by the National Science Foundation of china (NSFC) under grant 61372114 and 61571054 .

\section{References}

[1] J. Mitola, Q. Gerald and J. R. Maguire, "Cognitive radios: making software radios more Personal, IEEE Personal Communications, vol. 6, no. 4, (1999), pp. 13-18.

[2] P. Ubaidulla, H. Shin and M. Z. Win, "Robust wireless relay networks: slow power allocation with guaranteed GoS", IEEE Journal on Selected Topics in Signal Processing, vol. 1, no. 4, (2007), pp. 700-713.

[3] S. H. Safavi, M. Ardebilipour, S. Salari, "Relay beamforming in cognitive two-way networks with imperfect channel state information", IEEE Communications Letters, vol. 1, no. 4, (2012), pp. 344-347.

[4] T. Meixia and W. Rui, "Robust relay beamforming for two-way relay networks", IEEE Communications Letters, vol. 16, no. 7, (2012), pp. 1052-1055.

[5] H. Cai, Y. Wang and T. Yi, "An approach for minimizing aquadratically constrained fractional quadratic problem with application to the communications over wireless channels", Optimization method and software, vol. 29, no. 2, (2014), pp. 310-320.

[6] H. Cai and W. Ai, "Cooperative Beamforming Research for Two-Way Relayin Cognitive Radio by a New SDP Method", International Journal of Future Generation Communication and Networking, vol. 7, no. 1, (2014), pp. 159-168.

[7] R. A. Horn and C. Johnson, "Matrix Analysis". Cambridge University Press, (1985).

[8] Y. Y. Ye, "Linear conic programming, Work Paper", (2004), http://www.stanford.edu/class/msande314.

[9] S. Boyd and L. Vandenberghe, "Convex optimization", England: Cambridge University Press, (2004), p. 655.

[10] Y. W. Huang and S. Z. Zhang, "Approximation algorithms for indefinite complex quadratic maximization problems", Sci. China Ser. A, vol. 53, no. 10, (2010), pp. 2697-2708. 
International Journal of Future Generation Communication and Networking Vol. 9, No. 6 (2016) 